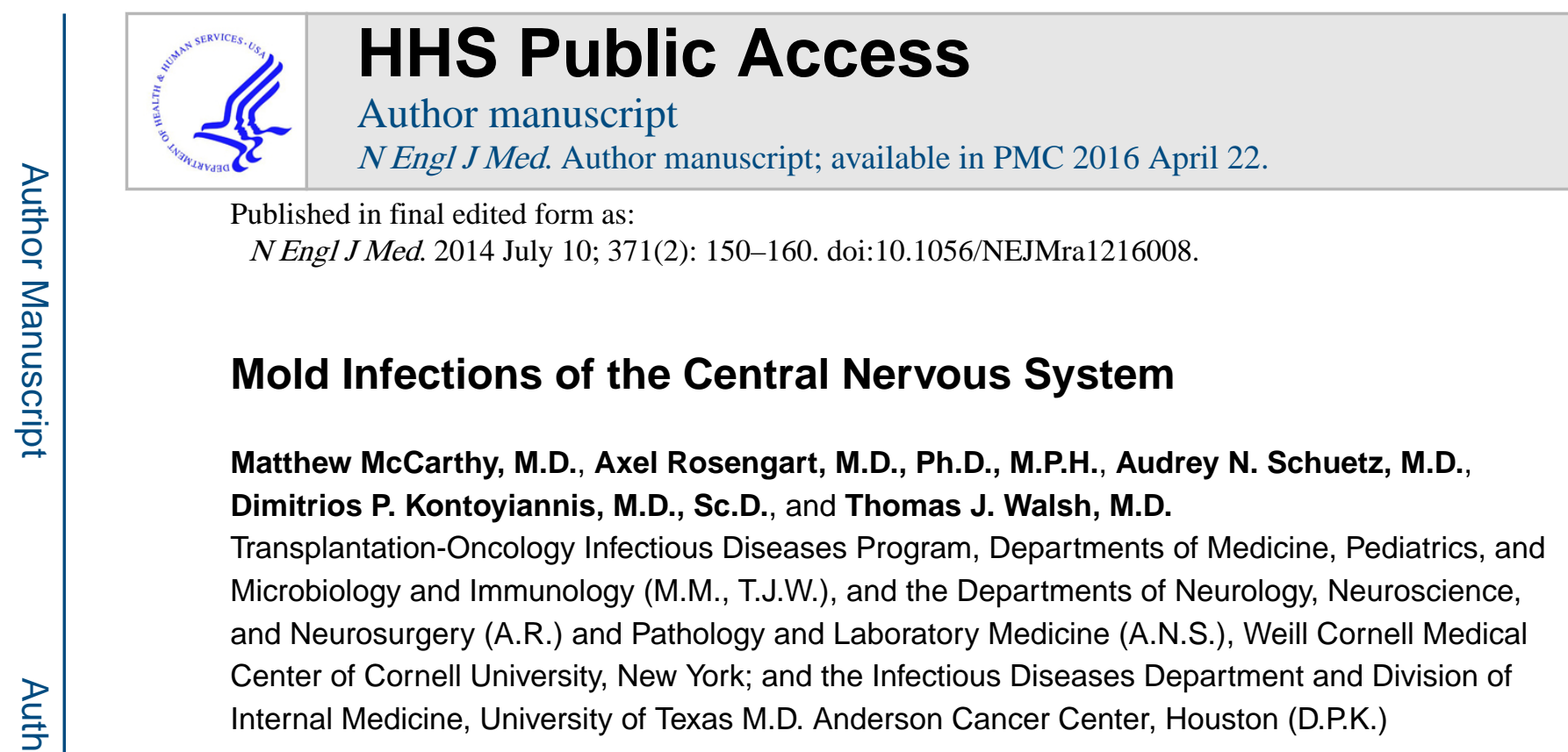

\title{
Abstract
}

The recent outbreak of exserohilum rostratum meningitis linked to epidural injections of methylprednisolone acetate has brought renewed attention to mold infections of the central nervous system (CNS). ${ }^{1}$ Although uncommon, these infections are often devastating and difficult to treat. This focused review of the epidemiologic aspects, clinical characteristics, and treatment of mold infections of the CNS covers a group of common pathogens: aspergillus, fusarium, and scedosporium species, molds in the order Mucorales, and dematiaceous molds. Infections caused by these pathogen groups have distinctive epidemiologic profiles, clinical manifestations, microbiologic characteristics, and therapeutic implications, all of which clinicians should understand.

\section{COMMON FEATURES}

Molds are ubiquitous organisms found in soil, water, and decaying vegetation. ${ }^{2}$ All have septate, angular, branching hyphae in tissue, with the exception of those in the order Mucorales, which have broad, ribbonlike, nonseptate or hyposeptate hyphae (Fig. 1). The respiratory tract is usually the portal of entry, with subsequent hematogenous dissemination to the CNS. However, direct inoculation of CNS or paraspinal tissue as a result of surgery, trauma, intravenous drug use, or contaminated medical supplies may also occur in immunocompetent persons, including those infected with Exophiala dermatitidis (formerly known as Wangiella dermatitidis) and E. rostratum. ${ }^{3}$ Organisms may also spread to the CNS from adjacent structures, including the sinuses, mastoid, and orbit. Infection of the ethmoid sinuses may lead to cavernous sinus thrombosis as a result of invasion of the emissary veins that drain the sinuses. Hyphae can invade from the ethmoid sinuses through the lamina

Address reprint requests to Dr. Walsh at the Transplantation-Oncology Infectious Diseases Program, Weill Cornell Medical Center of Cornell University, 1300 York Ave., Rm. A-421, New York, NY 10065, or at thw2003@med.cornell.edu.

Disclosure forms provided by the authors are available with the full text of this article at NEJM.org.

Dr. Kontoyiannis reports receiving fees for serving on an advisory board from Merck, personal fees from Gilead, and grant support from Merck, Pfizer, and Astellas. Dr. Walsh reports receiving fees for serving on advisory boards from Astellas, ContraFect, Pfizer, and iCo, consulting fees from Astellas, ContraFect, Pfizer, iCo, Methylgene, and grant support from Astellas, Novartis, ContraFect, Merck, and Cubist; he also reports serving as an uncompensated consultant for Drais, SigmaTau, and Trius, and receiving a study drug for laboratory investigation from Pfizer. No other potential conflict of interest relevant to this article was reported. 
papyracea and into the periorbital space, thus threatening the eye, extraocular muscles, and posterior apical structures, including the optic nerve.

Angioinvasion is common in immunocompromised patients and accounts for the hematogenous dissemination from the lungs that causes focal neurologic deficits (Fig. 2). Histopathological examination of involved brain tissue reveals hyphal angioinvasion with thrombosis in small and large vessels, hemorrhagic infarction, and coagulative necrosis, as well as vasculitis and granuloma formation. Susceptibility to angioinvasion also confers a predisposition to the formation of mycotic aneurysms. Without effective management, mold infections of the CNS carry poor prognoses, particularly in immunocompromised patients; such patients may present with isolated brain abscesses that simulate tumors of the CNS.

There are four cornerstones of the management of mold infections of the CNS: early diagnosis, administration of antifungal chemotherapy, neurosurgical assessment and intervention, and management of immunologic impairment. Figure 1 outlines the comparative epidemiologic profiles, clinical characteristics, laboratory features, and neuroimaging features of mold infections of the CNS. Early diagnosis may allow for timely therapeutic intervention and prevention of neurologic sequelae. Computed tomography (CT) and magnetic resonance imaging (MRI) are important adjuncts in the detection of infection and in monitoring the course of therapy. Patients who are at risk or who have documented invasive mold infection of the lungs or sinuses should be evaluated with the use of neuroimaging if they have any neurologic signs or symptoms. Patients with intracranial lesions suggestive of fungal abscesses or granulomas may be candidates for stereotactic biopsy.

Two distinct patterns characterize mold infections of the CNS. In some patients, the disease arises by direct extension from the paranasal sinuses, eye, or middle ear, causing a single abscess or a few abscesses. ${ }^{4,5}$ These lesions usually occur in the frontal or temporal lobe. Other patients have hematogenous infection, which may lead to solitary or multiple small abscesses that are often seen at the junction of cerebral gray and white matter and in the putamen-striatal arterial distribution. Mycotic aneurysms may form and rupture, which creates the potential for hemorrhagic strokes, subarachnoid hemorrhage, and empyema formation.

\section{EARLY DIAGNOSIS}

Detection of galactomannan antigen and 1,3- $\beta$-D-glucan in cerebrospinal fluid (CSF) may be helpful in establishing the diagnosis of CNS aspergillosis or other mold infections, such as fusariosis. ${ }^{6,7}$ However, because galactomannan antigen and 1,3- $\beta$-D-glucan can also be expressed by fusarium species, its presence in CSF does not constitute a definitive diagnosis of CNS aspergillosis ${ }^{8} ; 1,3-\beta$-D-glucan may also be expressed by scedosporium species and Exserohilum rostratum. A poly-merase-chain-reaction assay that is specific for aspergillus also may prove useful, but standardized platforms are lacking. ${ }^{9}$

\section{ANTIFUNGAL THERAPY}

Table 1 outlines therapeutic interventions for mold infections of the CNS. The arsenal against these infections includes three classes of antifungal agents: polyenes (amphotericin 
B formulations), triazoles (voriconazole, itraconazole, and posaconazole), and echinocandins (caspofungin, micafungin, and anidulafungin). Voriconazole is the primary agent for the treatment of CNS aspergillosis. ${ }^{12}$ Amphotericin B and its lipid formulations are first-line agents for the treatment of mucormycosis. The roles of other triazoles and echinocandins in the treatment of mold infections of the CNS have not been well defined.

Antifungal agents vary widely in their distribution in the CSF. ${ }^{13}$ Voriconazole is a relatively small (349-dalton), moderately lipophilic molecule with a concentration in CSF that is approximately $50 \%$ of its concentration in plasma, in both animals and humans. Other antimold triazoles, such as posaconazole and itraconazole (708 and 705 daltons, respectively), are highly lipophilic molecules with negligible concentrations in CSF. Amphotericin B (923 daltons) and its lipid formulations have relatively limited distribution in the CSF, but they do have detectable concentrations that are at or above inhibitory concentrations within brain parenchyma. Echinocandins are larger cyclic hexapeptide molecules (1140 to 1292 daltons) with relatively low or undetectable concentrations in CSF. The administration of antifungal agents through a ventriculostomy or by the intrathecal route is not recommended because of local toxic effects and a lack of proven efficacy.

\section{NEUROSURGICAL ASSESSMENT AND INTERVENTION}

The clinical characteristics of mold infections of the CNS warrant assessment for possible biopsy and neurosurgical intervention (Table 2). A definitive diagnosis almost invariably requires a biopsy, with prompt inspection of the specimen by means of wet-mount preparation with calcofluor white stain, culture, and histologic analysis (with Gomori methenamine silver stain and periodic acid-Schiff stain). In situ hybridization and immunohistochemical analysis may be helpful if cultures of biopsy specimens are negative. Because brain biopsies are highly invasive and may lead to neurologic deficits, they are often not feasible; therefore, diagnosis of a mold infection of the CNS is more often made by inference based on the recovery of the pathogen from a pulmonary or sinus source in a patient who has a CNS lesion that is radiologically consistent with a mold infection.

If the patient has a hemispheric ischemic stroke that is thought to be due to invasive fungal infection, and if the patient's clinical condition - that is, coexisting conditions, coagulation status, and overall health status - permits, early selective decompressive hemicraniectomy and durotomy may prevent devastating secondary brain herniation. Selective stereotactic decompression of edematous intracerebral lesions may prevent herniation while sparing functionally critical areas. Bulk excision of either intracranial abscesses or granulomas should be avoided, especially within critical functional regions.

Compressive and intramedullary spinal cord syndromes caused by intradural, extradural, or vertebral fungal infections necessitate prompt neurosurgical decompression, drainage, and resection. Surgical drainage or excision, tailored to the location and size of the lesion, is the first-line treatment for intramedullary abscess or granuloma formations. In contrast, localized meningitis, arachnoiditis, radiculopathy, radiculomyelopathy, and anterior-spinalartery infarction are treated conservatively. 


\section{MANAGEMENT OF IMMUNOLOGIC IMPAIRMENTS}

Because innate host defenses play a critical role in protection from and eradication of mold infections of the CNS, the reversal of immunosuppression is essential for a successful outcome. The intrinsic immune responses within the brain, involving microglia and the complement system, appear to be inadequate as a host defense against invasive Aspergillus fumigatus. ${ }^{14}$ In transplant recipients, for whom reversal of immunosuppression may not be feasible, immunosuppressive therapy should be minimized in close coordination with the patient's primary care team.

Strategies that can augment the innate host response against mold infection of the CNS include the use of growth factors to accelerate recovery from neutropenia, provision of granulocyte transfusions with sustained circulating neutrophils until the patient recovers from neutropenia, and discontinuation or reduction in the dose of glucocorticoids. ${ }^{15,16}$ The correction of metabolic acidosis and hyperglycemia is a cornerstone of the treatment of CNS mucormycosis.

\section{SPECIFIC PATHOGENS}

The management of mold infections of the CNS is individualized for each patient on the basis of the host response, neuroanatomical involvement, and the specific pathogen involved.

\section{ASPERGILLUS SPECIES}

The risk factors for CNS aspergillosis include neutropenia, systemic glucocorticoid treatment, mastoidectomy, spinal anesthesia, and paraspinal glucocorticoid injections. ${ }^{17} \mathrm{~A}$. fumigatus was isolated from the index patient in the 2012 outbreak of fungal meningitis. ${ }^{18}$

Focal neurologic deficits and seizures caused by stroke or mass effect are the most common clinical manifestations of CNS aspergillosis. ${ }^{19}$ Meningeal signs are uncommon, and their presence is indicative of a subarachnoid hemorrhage. ${ }^{20} \mathrm{CNS}$ aspergillosis should be high on the list of disorders in the differential diagnosis for patients with immunosuppression and focal brain lesions, especially those with characteristic pulmonary infiltrates in whom focal neurologic deficits or focal seizures develop. Recovery of aspergillus from pulmonary lesions with the use of bronchoalveolar lavage or fine-needle aspiration should be pursued when possible. An enzyme immunoassay for detection of galactomannan in serum or bronchoalveolar lavage fluid should be performed when feasible. ${ }^{21}$ As described above, galactomannan and 1,3- $\beta$-D-glucan may be found in the serum or CSF of patients with CNS aspergillosis. 6,7

Voriconazole is the first-line treatment for CNS aspergillosis. ${ }^{12}$ Although there are no formal guidelines regarding therapeutic drug monitoring in CNS aspergillosis, we recommend maintaining trough concentrations of 2 to $5 \mu \mathrm{g}$ per milliliter in serum. Drug-related adverse events may necessitate a reduction in dosage. Because voriconazole has approximately $50 \%$ penetration through the blood-brain barrier, measurement of concentrations in CSF is not necessary. For patients in whom voriconazole as primary therapy might have unacceptable adverse effects, liposomal amphotericin B is an alternative. ${ }^{12}$ Monitoring of the therapeutic response of CNS aspergillosis and other mold infections of the CNS should include careful 
bedside evaluation and serial CT or MRI scans. The frequency of scanning should vary directly with the severity of the infection, but a reasonable interval is a minimum of every 1 to 2 weeks until the patient's condition is stable.

\section{MUCORALES}

Cerebral mucormycosis, which is perhaps the most aggressive mold infection of the CNS, constitutes a medical emergency. Early cases were uniformly fatal. However, recent studies have shown improved survival, possibly as the result of earlier diagnosis and better control of underlying diseases. ${ }^{22}$ Diabetes mellitus and iron-overload conditions are distinctive risk factors for the development of mucormycosis. ${ }^{23}$ In patients with neutropenia or patients receiving glucocorticoid therapy, mold infections of the CNS develop as sino-orbital infections or through hematogenous dissemination of pulmonary mucormycosis. ${ }^{24}$ In contrast, patients with diabetes mellitus usually present with sino-orbital mucormycosis and seldom present with pulmonary or disseminated infection. ${ }^{23,25}$ Among intravenous drug users, CNS mucormycosis is a relatively common cause of intracerebral fungal abscesses. ${ }^{25}$ Treatment with the iron-chelating agent deferoxamine is a well-recognized risk factor for disseminated mucormycosis. ${ }^{26}$

Perhaps more than any other infection, mucormycosis of the ethmoid sinuses may involve all structures along its invasive path, including the orbit and eye, bone, and brain tissue. ${ }^{25,27}$ Because venous drainage of the ethmoid sinuses extends into the cavernous sinuses, ethmoidal mucormycosis carries a high risk of cavernous sinus thrombosis. Impairment of cranial nerves III, IV, $\mathrm{V}_{1}, \mathrm{~V}_{2}$, and VI may be the initial signs of cavernous sinus thrombosis. Sinus opacification, bony erosions, and obliteration of deep fascia planes can be detected with the use of CT and MRI. The organism may be identified by swabbing, scraping, or biopsy of an involved area to obtain samples for analysis, such as "black pus" from the nasal turbinates, fluid from a surgically drained sinus, or brain tissue. Wet-mount preparations reveal ribbonlike, broad, nonseptate or hyposeptate hyphae, which are highlighted with the use of calcofluor white. Because it can be difficult to detect hyphae in frozen sections stained with hematoxylin and eosin, some institutions have introduced calcofluor-stained homogenates of intraoperative specimens as a rapid means of determining whether Mucorales organisms are present. ${ }^{28}$

Successful management of rhinocerebral mucormycosis depends not only on early diagnosis but also on primary antifungal therapy with amphotericin B, reversal of host impairments particularly diabetes mellitus, neutropenia, and glucocorticoid exposure — and timely surgical intervention, when indicated. Posaconazole, which has in vitro activity against some isolates of Mucorales, particularly species of mucor, may have a role in follow-up therapy. ${ }^{29}$ Although iron chelation with deferasirox has been shown to improve the outcome in a diabetic murine model of disseminated mucormycosis, a randomized trial of this agent did not show a benefit. ${ }^{30}$ Adjunctive treatments, such as hyper-baric oxygen, granulocytemacrophage colony-stimulating factor, and interferon- $\gamma$, require further study. ${ }^{31}$ 


\section{FUSARIUM SPECIES}

CNS fusariosis develops predominantly in patients with prolonged neutropenia. These organisms are highly angioinvasive and cause hemorrhagic infarction with strokelike events. ${ }^{32}$ Portals of entry include the lungs, sinuses, vascular catheters, and distinctively, periungual lesions (paronychia in patients with neutropenia). ${ }^{33}$ Fusarium species are also most frequently associated with fungemia, multiple erythematous nodular cutaneous lesions, and septic arthritis. A positive blood culture growing a hyaline (i.e., colorless or lightly pigmented) mold in a patient with persistent neutropenia is most likely to indicate the presence of fusarium. A definitive mycologic diagnosis can be rapidly established by biopsy and culture of these cutaneous lesions. As compared with other mold infections of the CNS, disseminated fusariosis is more commonly associated with bilateral endophthalmitis, which may lead to blindness. Hematogenously disseminated fusarium infections cause chorioretinitis at a rate that is disproportionally higher than that seen with other organisms causing mold infections of the CNS. ${ }^{34}$ Because disseminated fusariosis commonly causes fungemia and cutaneous lesions, early diagnosis and therapy may prevent progression to encephalitis and endophthalmitis.

Fusarium species vary in their susceptibility to antifungal agents. ${ }^{35}$ Voriconazole is licensed for second-line therapy; however, amphotericin B also has been used successfully. ${ }^{36} \mathrm{~A}$ rational approach for treating patients with CNS fusariosis, pending determination of susceptibility, is the administration of voriconazole plus a lipid formulation of amphotericin B.

\section{SCEDOSPORIUM SPECIES}

Scedosporium apiospermum is readily isolated from soil, polluted water, and sewage. Mold infections of the CNS caused by $S$. apiospermum are strongly associated with drowning events in immunocompetent hosts, when the organism invades through the cribriform plate or disseminates from pneumonic foci. ${ }^{37,38}$ Among immunocompromised patients, the features of CNS scedosporiosis resemble those of aspergillosis, including hematogenous dissemination from pulmonary lesions or by extension from the sinuses. Although scedosporium species form branching septate hyphae that are similar to those of aspergillus, in rare cases, terminal annelloconidia are seen in tissue. Nonetheless, culture is required for a definitive diagnosis. Voriconazole is licensed as second-line therapy for $S$. apiospermum infections and, in the absence of alternatives, is used as initial therapy, until the in vitro susceptibility profile is available. ${ }^{39}$

In contrast, $S$. prolificans is resistant experimentally and clinically to the three major classes of antifungal agents. S. prolificans infects the CNS through hematogenous dissemination in immunocompromised patients and through traumatic inoculation in immunocompetent hosts. ${ }^{37}$ Unlike $S$. apiospermum, $S$. prolificans may be recovered from blood cultures. Therapy consists of surgical resection and reversal of immunosuppression.

\section{DEMATIACEOUS MOLDS}

Dematiaceous fungi are a group of molds characterized by the presence of melanin-like pigment within the cell wall that is pale brown to black. ${ }^{40}$ These organisms can be pathogens 
to plants or livestock and may cause chromoblastomycosis and black-grain mycetoma. Dematiaceous molds may also cause deeply invasive infections (phaeo-hyphomycosis), including infections of the CNS. Most of the agents causing phaeohyphomycosis grow very slowly, so classic mycologic identification can take 3 weeks or longer. In comparison with aspergillus, Mucorales, and fusarium, dematiaceous molds commonly cause infections of the CNS in immunocompetent hosts. Some dematiaceous molds within a narrow geographic range cause cerebral phaeohyphomycosis. ${ }^{41}$

Until October 2012, most known cases of CNS phaeohyphomycosis were caused by Cladophialophora bantiana (also called Xylohypha bantiana), Rhinocladiella mackenziei, and Ochroconis gallopava (also called Dactylaria gallopava). ${ }^{40}$ E. rostratum has since emerged as a cause of meningitis in a nationwide outbreak associated with tainted epidural glucocorticoid injections. ${ }^{42,43}$ Prolonged receipt of antifungal chemotherapy, surgical resection of abscesses, and reversal of immunosuppression are mainstays of the treatment of CNS phaeohyphomycosis. Persistence or recurrence of disease is common.

Mold infections of the CNS caused by $C$. bantiana are manifested as a slowly expanding, space-occupying lesion causing headache, seizure, and localizing neurologic signs that simulate a brain tumor. ${ }^{40}$ Among immunocompetent patients, CNS infection may occur in the absence of pulmonary lesions. Patients who have survived had easily resectable, encapsulated masses on CT or MRI scans, whereas those who have died had a solitary lesion that was not entirely resected, poorly demarcated abscess borders, or multiple satellite lesions. ${ }^{44}$ There is no clearly effective antifungal therapy. ${ }^{45}$ Surgical resection remains the most definitive treatment.

O. gallopava is a neurotropic dematiaceous mold that causes pulmonary and CNS infection in domestic poultry and in immunocompromised humans. ${ }^{46}$ Exposure to infested aerosolized warm water may be a risk factor. In one study involving transplant recipients, the CNS was involved in $50 \%$ of patients (6 of 12) with O. gallopava infection and a poor outcome. ${ }^{47}$ The most effective antifungal regimen for treatment of this infection is not known. Interpretive breakpoints for in vitro antifungal susceptibility tests have not been established for this organism. Combination antifungal therapy with voriconazole and a lipid formulation of amphotericin B, pending the availability of in vitro susceptibility data, is recommended in conjunction with surgery and reversal of immunosuppression.

E. rostratum is an opportunistic mold that until recently was an uncommon cause of disease in immunocompromised and immunocompetent human hosts. ${ }^{48}$ On September 18, 2012, health officials began to react to a large, multistate outbreak of fungal meningitis traceable to three lots of preservative-free methylprednisolone from one compounding pharmacy in Massachusetts. This outbreak resulted in 751 cases of CNS infection and 64 deaths across the United States. Three molecular assays and assays for 1,3- $\beta$-D-glucan in serum and CSF have been developed and may serve as adjunctive diagnostic tools. ${ }^{49-52}$ Most of the infected patients in the outbreak presented with signs and symptoms that were consistent with fungal meningitis; however, cases of spinal osteomyelitis or epidural abscess and septic arthritis or osteomyelitis were also reported. ${ }^{1}$ In vitro susceptibility data indicate that voriconazole and amphotericin B have activity against $E$. rostratum; this has led to recommendations that 
voriconazole, liposomal amphotericin $\mathrm{B}$, or both be used for initial management in conjunction with neurosurgical consultation, when appropriate. ${ }^{42,43}$ A more detailed review of the management of $E$. rostratum meningitis is reported elsewhere. ${ }^{42,53}$ A comparison of the clinical features of less common mold infections of the CNS is provided in Table S1 of the Supplementary Appendix, available with the full text of this article at NEJM.org.

\section{Supplementary Material}

Refer to Web version on PubMed Central for supplementary material.

\section{References}

1. Smith RM, Schaefer MK, Kainer MA, et al. Fungal infections associated with contaminated methylprednisolone injections. N Engl J Med. 2013; 369:1598-609. [PubMed: 23252499]

2. Segal BH. Aspergillosis. N Engl J Med. 2009; 360:1870-84. [PubMed: 19403905]

3. Perfect JR. Iatrogenic fungal meningitis: tragedy repeated. Ann Intern Med. 2012; 157:825-6. [PubMed: 23080380]

4. Walsh TJ, Hier DB, Caplan LR. Fungal infections of the central nervous system: comparative analysis of risk factors and clinical signs in 57 patients. Neurology. 1985; 35:1654-7. [PubMed: 4058755]

5. Li DM, de Hoog GS. Cerebral phaeohyphomycosis - a cure at what lengths? Lancet Infect Dis. 2009; 9:376-83. [PubMed: 19467477]

6. Soeffker G, Wichmann D, Loderstaedt U, Sobottka I, Deuse T, Kluge S. Aspergillus galactomannan antigen for diagnosis and treatment monitoring in cerebral aspergillosis. Prog Transplant. 2013; 23:71-4. [PubMed: 23448824]

7. Mikulska M, Furfaro E, Del Bono V, et al. (1-3)- $\beta$-D-glucan in cerebrospinal fluid is useful for the diagnosis of central nervous system fungal infections. Clin Infect Dis. 2013; 56:1511-12. [PubMed: 23392391]

8. Tortorano AM, Esposto MC, Prigitano A, et al. Cross-reactivity of Fusarium spp. in the Aspergillus galactomannan enzyme-linked immunosorbent assay. J Clin Microbiol. 2012; 50:1051-3. [PubMed: 22205818]

9. Reinwald M, Buchheidt D, Hummel M, et al. Diagnostic performance of an Aspergillus-specific nested PCR assay in cerebrospinal fluid samples of immunocompromised patients for detection of central nervous system aspergillosis. PLoS One. 2013; 8(2):e56706. [PubMed: 23451071]

10. Johnson EM, Szekely A, Warnock DW. In-vitro activity of voriconazole, itraconazole and amphotericin B against filamentous fungi. J Antimicrob Chemother. 1998; 42:741-5. [PubMed: 10052897]

11. Pfaller MA, Espinel-Ingroff A, Canton E, et al. Wild-type MIC distributions and epidemiological cutoff values for amphotericin B, flucytosine, and itraconazole and Candida spp. as determined by CLSI broth microdilution. J Clin Microbiol. 2012; 50:2040-6. [PubMed: 22461672]

12. Walsh TJ, Anaissie EJ, Denning DW, et al. Treatment of aspergillosis: clinical practice guidelines of the Infectious Diseases Society of America. Clin Infect Dis. 2008; 46:327-60. [PubMed: 18177225]

13. Schwartz S, Thiel E. Cerebral aspergillosis: tissue penetration is the key. Med Mycol. 2009; 47(Suppl 1):S387-S393. [PubMed: 19255905]

14. Rambach G, Hagleitner M, Mohsenipour I, et al. Antifungal activity of the local complement system in cerebral aspergillosis. Microbes Infect. 2005; 7:1285-95. [PubMed: 16027023]

15. Ben-Ami R, Lewis RE, Raad II, Kontoyiannis DP. Phaeohyphomycosis in a tertiary care cancer center. Clin Infect Dis. 2009; 48:1033-41. [PubMed: 19267655]

16. Katragkou A, Roilides E. Immunotherapy of infections caused by rare filamentous fungi. Clin Microbiol Infect. 2012; 18:134-9. [PubMed: 22044625] 
17. Antinori S, Corbellino M, Meroni L, et al. Aspergillus meningitis: a rare clinical manifestation of central nervous system aspergillosis: case report and review of 92 cases. J Infect. 2013; 66:218-38. [PubMed: 23178421]

18. Pettit AC, Kropski JA, Castilho JL, et al. The index case for the fungal meningitis outbreak in the United States. N Engl J Med. 2012; 367:2119-25. [PubMed: 23083311]

19. Kourkoumpetis TK, Desalermos A, Muhammed M, Mylonakis E. Central nervous system aspergillosis: a series of 14 cases from a general hospital and review of 123 cases from the literature. Medicine (Baltimore). 2012; 91:328-36. [PubMed: 23117848]

20. Iihara K, Makita Y, Nabeshima S, Tei T, Keyaki A, Nioka H. Aspergillosis of the central nervous system causing subarachnoid hemorrhage from mycotic aneurysm of the basilar artery - case report. Neurol Med Chir (Tokyo). 1990; 30:618-23. [PubMed: 1703641]

21. Herbrecht R, Letscher-Bru V, Oprea C, et al. Aspergillus galactomannan detection in the diagnosis of invasive aspergillosis in cancer patients. J Clin Oncol. 2002; 20:1898-906. [PubMed: 11919250]

22. Hammond SP, Baden LR, Marty FM. Mortality in hematologic malignancy and hematopoietic stem cell transplant patients with mucormycosis, 2001 to 2009. Antimicrob Agents Chemother. 2011; 55:5018-21. [PubMed: 21876046]

23. Mantadakis E, Samonis G. Clinical presentation of zygomycosis. Clin Microbiol Infect. 2009; 15(Suppl 5):15-20. [PubMed: 19754751]

24. McNulty JS. Rhinocerebral mucormycosis: predisposing factors. Laryngoscope. 1982; 92:1140-3. [PubMed: 7132514]

25. Hopkins RJ, Rothman M, Fiore A, Goldblum SE. Cerebral mucormycosis associated with intravenous drug use: three case reports and review. Clin Infect Dis. 1994; 19:1133-7. [PubMed: 7888545]

26. Ibrahim AS, Spellberg B, Walsh TJ, Kontoyiannis DP. Pathogenesis of mucormycosis. Clin Infect Dis. 2012; 54(Suppl 1):S16-S22. [PubMed: 22247441]

27. Petrikkos G, Skiada A, Lortholary O, Roilides E, Walsh TJ, Kontoyiannis DP. Epidemiology and clinical manifestations of mucormycosis. Clin Infect Dis. 2012; 54(Suppl 1):S23-S34. [PubMed: 22247442]

28. McDermott NE, Barrett J, Hipp J, et al. Successful treatment of periodontal mucormycosis: report of a case and literature review. Oral Surg Oral Med Oral Pathol Oral Radiol Endod. 2010; 109(3):e64-e69. [PubMed: 20219588]

29. Greenberg RN, Mullane K, van Burik JA, et al. Posaconazole as salvage therapy for zygomycosis. Antimicrob Agents Chemother. 2006; 50:126-33. [PubMed: 16377677]

30. Spellberg B, Ibrahim AS, Chin-Hong PV, et al. The Deferasirox-AmBisome Therapy for Mucormycosis (DEFEAT Mucor) study: a randomized, double-blinded, placebo-controlled trial. J Antimicrob Chemother. 2012; 67:715-22. [PubMed: 21937481]

31. Spellberg B, Ibrahim A, Roilides E, et al. Combination therapy for mucormycosis: why, what, and how? Clin Infect Dis. 2012; 54(Suppl 1):S73-S78. [PubMed: 22247449]

32. Martino P, Gastaldi R, Raccah R, Girmenia C. Clinical patterns of Fusarium infections in immunocompromised patients. J Infect. 1994; 28(Suppl 1):7-15. [PubMed: 8077692]

33. Steinberg GK, Britt RH, Enzmann DR, Finlay JL, Arvin AM. Fusarium brain abscess: case report. J Neurosurg. 1983; 58:598-601. [PubMed: 6827355]

34. Lamaris GA, Esmaeli B, Chamilos G, et al. Fungal endophthalmitis in a tertiary care cancer center: a review of 23 cases. Eur J Clin Microbiol Infect Dis. 2008; 27:343-7. [PubMed: 18183439]

35. Anaissie E, Paetznick V, Proffitt R, Adler-Moore J, Bodey GP. Comparison of the in vitro antifungal activity of free and liposome-encapsulated amphotericin B. Eur J Clin Microbiol Infect Dis. 1991; 10:665-8. [PubMed: 1748123]

36. Dignani MC, Anaissie E. Human fusariosis. Clin Microbiol Infect. 2004; 10(Suppl 1):67-75. [PubMed: 14748803]

37. Cortez KJ, Roilides E, Quiroz-Telles F, et al. Infections caused by Scedosporium spp. Clin Microbiol Rev. 2008; 21:157-97. [PubMed: 18202441]

38. Montejo M, Muñiz ML, Zárraga S, et al. Case reports: infection due to Scedosporium apiospermum in renal transplant recipients: a report of two cases and literature review of central 
nervous system and cutaneous infections by Pseudallescheria boydii/Sc. apiospermum. Mycoses. 2002; 45:418-27. [PubMed: 12421295]

39. Troke P, Aguirrebengoa K, Arteaga C, et al. Treatment of scedosporiosis with voriconazole: clinical experience with 107 patients. Antimicrob Agents Chemother. 2008; 52:1743-50. [PubMed: 18212110]

40. Revankar SG, Sutton DA, Rinaldi MG. Primary central nervous system phaeohyphomycosis: a review of 101 cases. Clin Infect Dis. 2004; 38:206-16. [PubMed: 14699452]

41. Al-Tawfiq JA, Boukhamseen A. Cerebral phaeohyphomycosis due to Rhinocladiella mackenziei (formerly Ramichloridium mackenziei): case presentation and literature review. J Infect Public Health. 2011; 4:96-102. [PubMed: 21663879]

42. Kauffman CA, Pappas PG, Patterson TF. Fungal infections associated with contaminated methylprednisolone injections. N Engl J Med. 2013; 368:2495-500. [PubMed: 23083312]

43. Kainer MA, Reagan DR, Nguyen DB, et al. Fungal infections associated with contaminated methylprednisolone in Tennessee. N Engl J Med. 2012; 367:2194-203. [PubMed: 23131029]

44. Dixon DM, Walsh TJ, Merz WG, Mc-Ginnis MR. Infections due to Xylohypha bantiana (Cladosporium trichoides). Rev Infect Dis. 1989; 11:515-25. [PubMed: 2672237]

45. Lyons MK, Blair JE, Leslie KO. Successful treatment with voriconazole of fungal cerebral abscess due to Cladophialophora bantiana. Clin Neurol Neurosurg. 2005; 107:532-4. [PubMed: 16202830]

46. Qureshi ZA, Kwak EJ, Nguyen MH, Silveira FP. Ochroconis gallopava: a dematiaceous mold causing infections in transplant recipients. Clin Transplant. 2012; 26:E17-E23. [PubMed: 21955216]

47. Shoham S, Pic-Aluas L, Taylor J, Cortez K, et al. Transplant-associated Ochroconis gallopava infections. Transpl Infect Dis. 2008; 10:442-8. [PubMed: 18651872]

48. Stevens DA. Reflections on the approach to treatment of a mycologic disaster. Antimicrob Agents Chemother. 2013; 57:1567-72. [PubMed: 23384533]

49. Gade L, Scheel CM, Pham CD, et al. Detection of fungal DNA in human body fluids and tissues during a multistate outbreak of fungal meningitis and other infections. Eukaryot Cell. 2013; 12:677-83. [PubMed: 23457192]

50. Zhao Y, Petraitiene R, Walsh TJ, Perlin DS. A real-time PCR assay for rapid detection and quantification of Exserohilum rostratum, a causative pathogen of fungal meningitis associated with injection of contaminated methylprednisolone. J Clin Microbiol. 2013; 51:1034-6. [PubMed: 23303500]

51. Lyons JL, Roos KL, Marr KA, et al. Cerebrospinal fluid (1,3)- $\beta$-D-glucan detection as an aid for diagnosis of iatrogenic fungal meningitis. J Clin Microbiol. 2013; 51:1285-7. [PubMed: 23363831]

52. Litvintseva AP, Lindsley MD, Gade L, et al. Utility of (1-3)- $\beta$-D-glucan testing for diagnostics and monitoring response to treatment during the multistate outbreak of fungal meningitis and other infections. Clin Infect Dis. 2014; 58:622-30. [PubMed: 24336827]

53. Kontoyiannis DP, Perlin DS, Roilides E, Walsh TJ. What can we learn and what do we need to know amidst the iatrogenic outbreak of Exserohilum rostratum meningitis? Clin Infect Dis. 2013; 57:853-9. [PubMed: 23650291] 


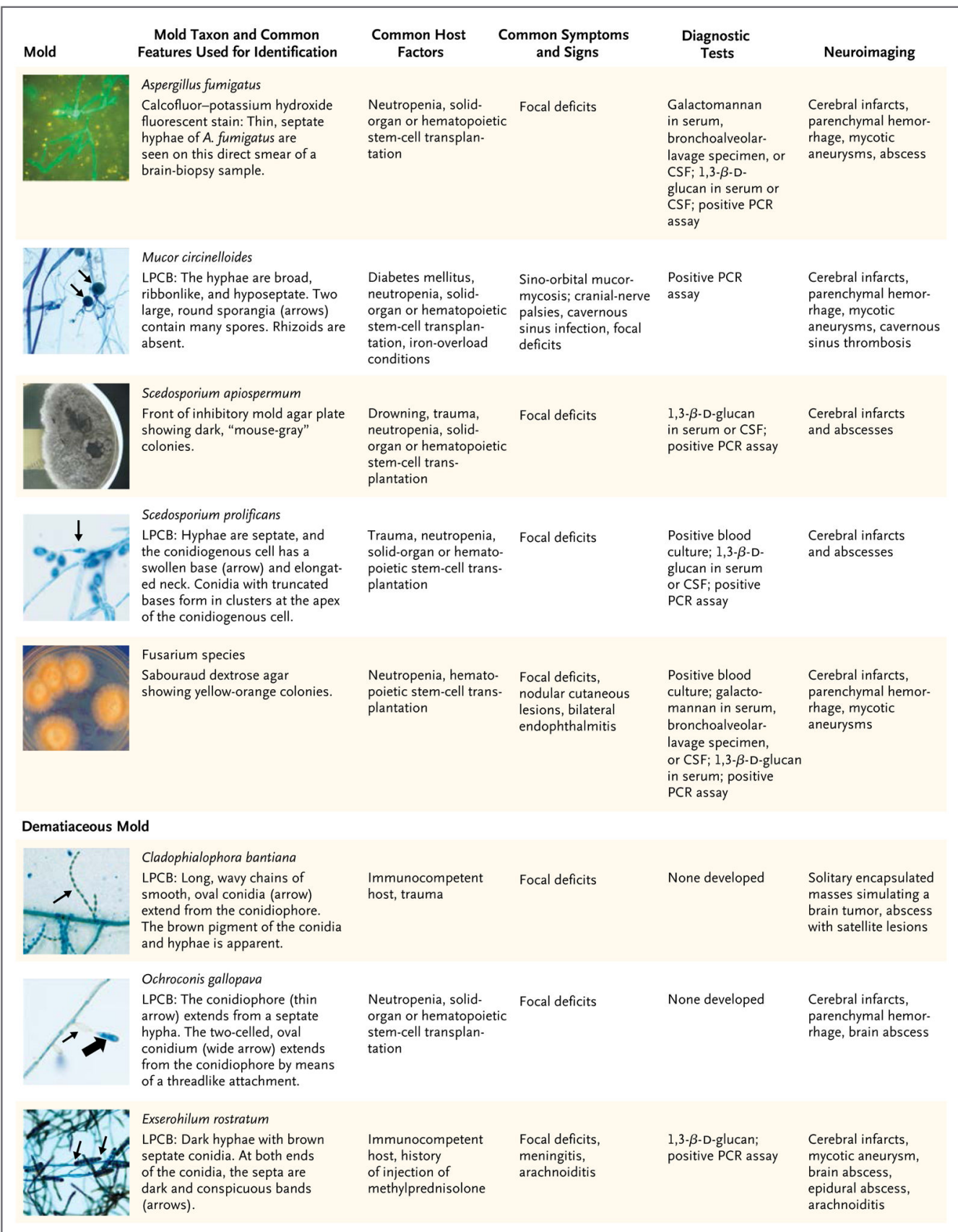

Figure 1. Comparative Features of Mold Infections of the Central Nervous System

The use of a polymerase-chain-reaction (PCR) assay of cerebrospinal fluid (CSF) remains investigational and has not been approved by the Food and Drug Administration. The host factors listed here are the most common; however, other hosts may be susceptible. LPCB denotes lacto-phenol cotton blue stain. 

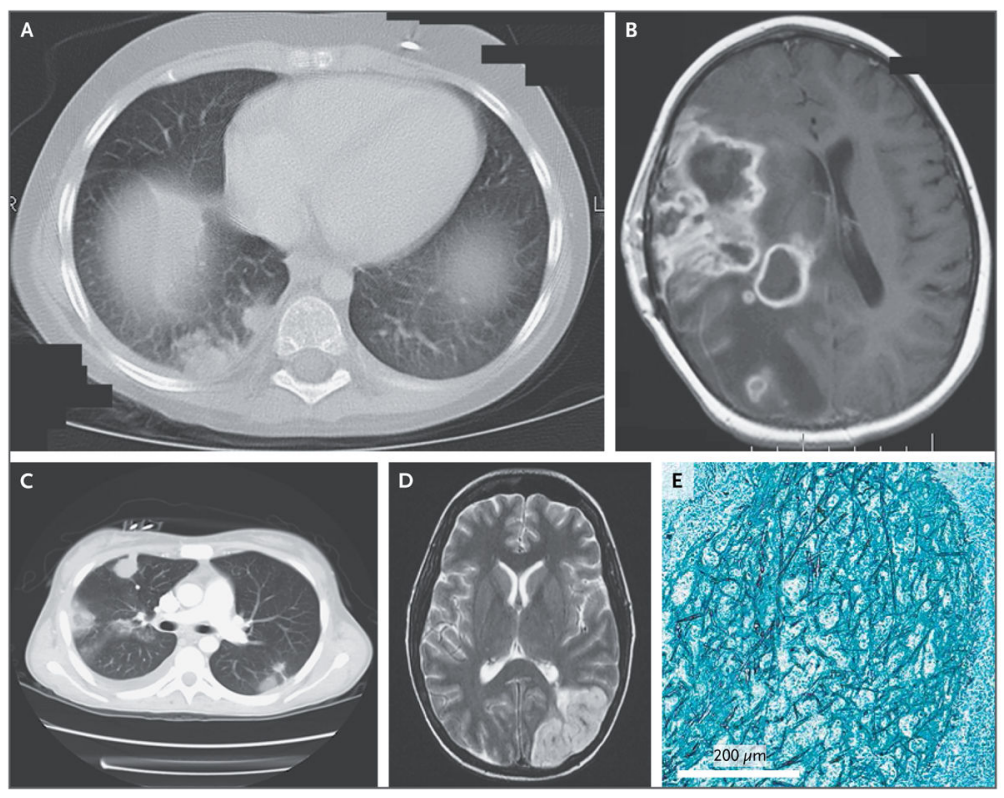

Figure 2. Characteristic Findings Associated with Mold Infections of the Central Nervous System

Patient 1, a 7-year-old girl with newly diagnosed, high-risk, pre-B-cell acute lymphocytic leukemia, presented with neutropenia and cough, paralysis of the left arm, and left homonymous hemianopsia. Computed tomography (CT) of the chest (Panel A) revealed nodular densities in the lower lobe of the right lung. Magnetic resonance imaging (MRI) of the head (Panel B) showed numerous cortical and subcortical infarcts with surrounding edema leading to mass effects; stereotactically guided brain biopsy yielded a specimen for culture that was positive for Aspergillus fumigatus. Patient 2, a 24-year-old woman with acute myeloid leukemia, was admitted for fever and neutropenia followed by rapid deterioration of mental status; CT of the chest (Panel C) showed nodular pneumonia. MRI of the head (Panel D) revealed multiple bilateral infarcts, the largest of which shown in the left parietal-occipital region. Examination of a brain-biopsy specimen (Panel E, Gomori methenamine silver stain) revealed numerous acutely branching septate hyphae. No organisms were grown in culture, because the biopsy specimen was obtained while the patient was receiving antifungal therapy. The histologically compatible organisms include aspergillus, fusarium, scedosporium, and other hyaline molds. The use of immunohistochemical and molecular diagnostic procedures, such as a PCR assay and in situ hybridization, may make it possible to further identify the causative organism. 


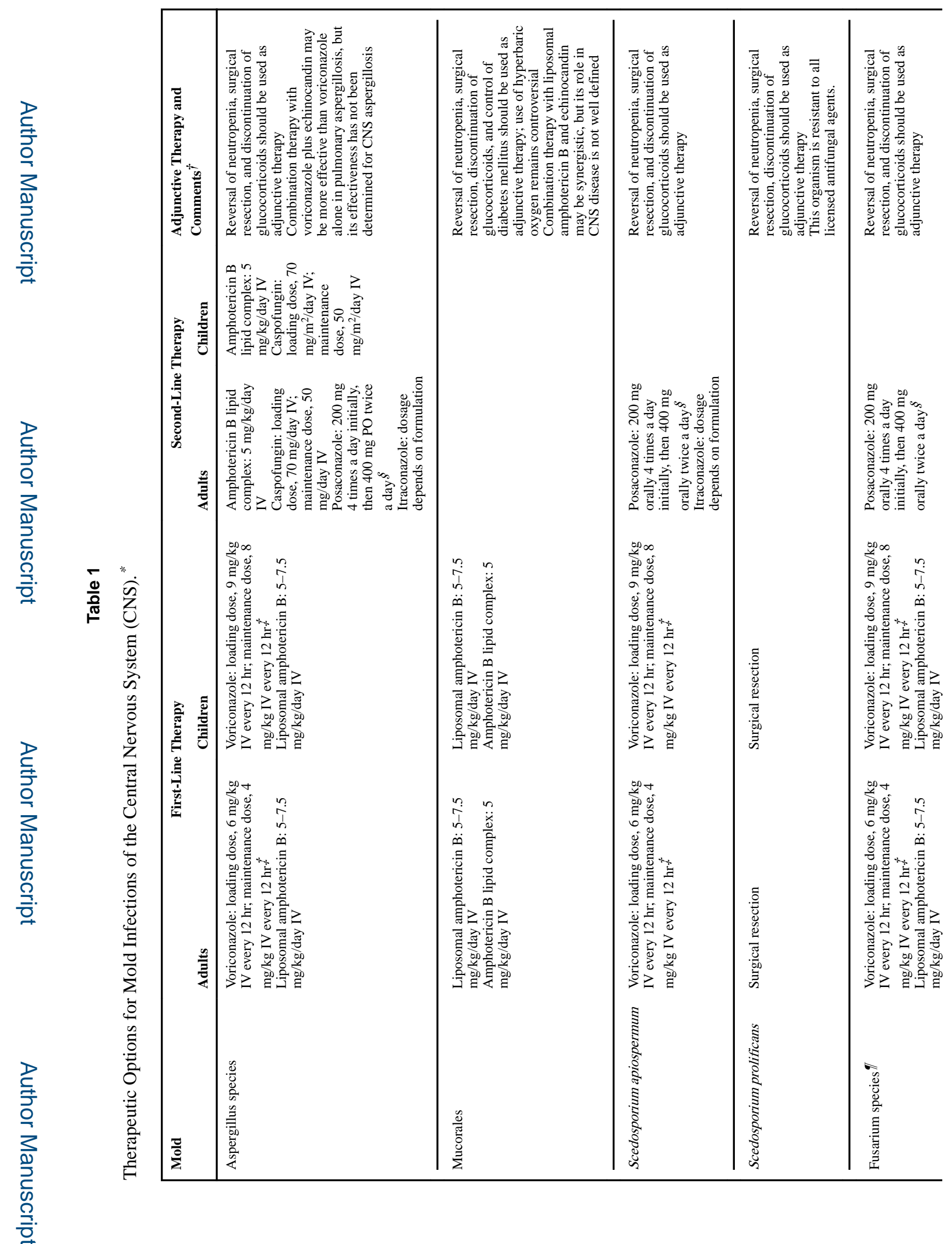

N Engl J Med. Author manuscript; available in PMC 2016 April 22. 


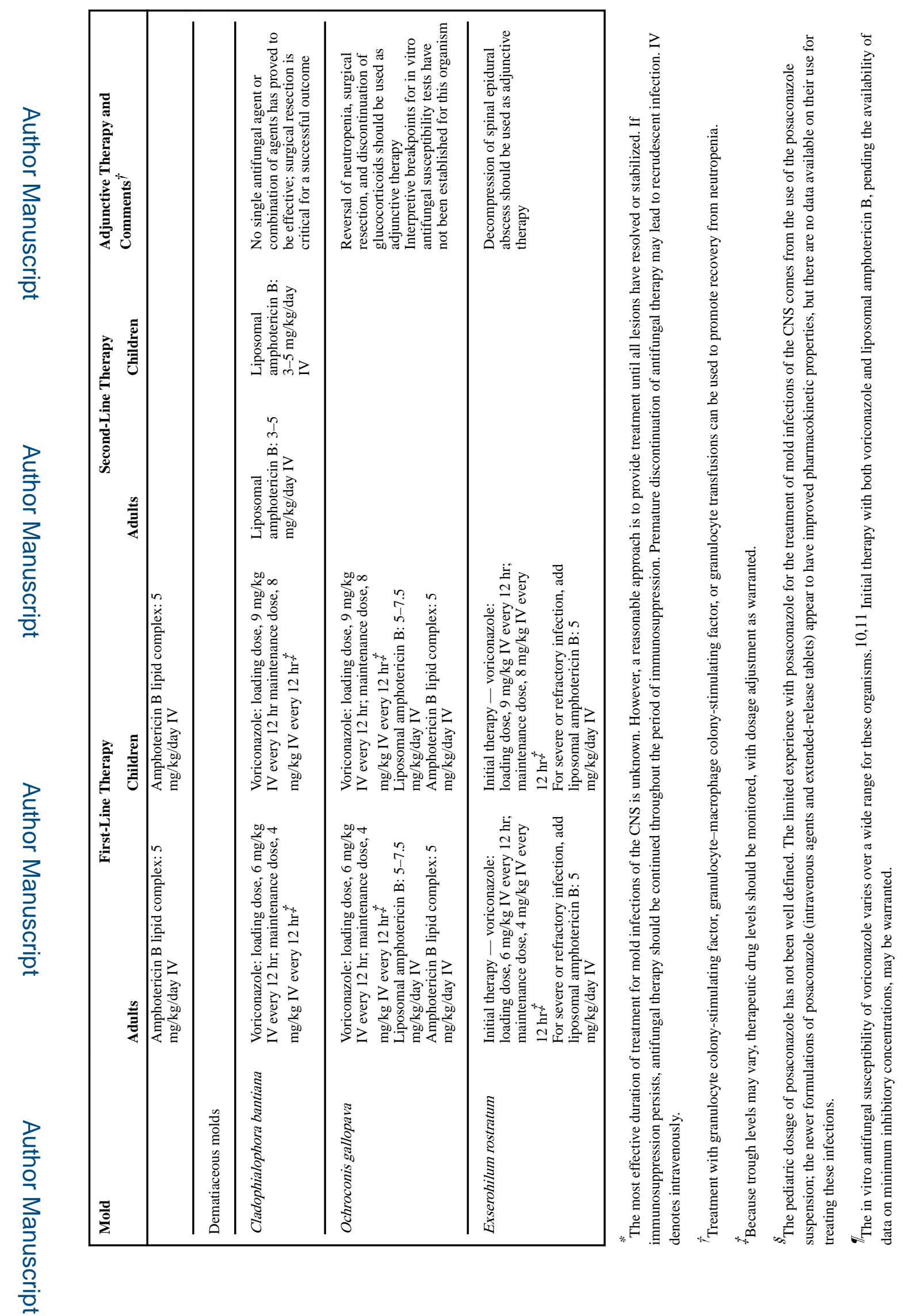

NEngl J Med. Author manuscript; available in PMC 2016 April 22. 
Table 2

Criteria for Neurosurgical or Neuroradiologic Intervention.

\begin{tabular}{|c|c|c|}
\hline Indication & Intervention & Comments \\
\hline $\begin{array}{l}\text { CNS space-occupying lesion } \\
\text { with mass effects }\end{array}$ & $\begin{array}{l}\text { Surgical decompression with } \\
\text { debulking of lesion or stereotactic } \\
\text { drainage }\end{array}$ & $\begin{array}{l}\text { Choice of approach depends on lesion size, location, and severity; } \\
\text { large masses with evidence of brain or spinal cord compression or } \\
\text { herniation syndromes (with or without increases in intracranial } \\
\text { pressure) should immediately be decompressed, especially in } \\
\text { patients with a rapid onset of clinical deterioration }\end{array}$ \\
\hline Hydrocephalus & $\begin{array}{l}\text { Insertion of extraventricular drainage } \\
\text { catheter }\end{array}$ & $\begin{array}{l}\text { Clinical deterioration with rostrocaudal brain herniation can occur } \\
\text { rapidly as a result of unrecognized hydrocephalus; an external } \\
\text { ventricular drain allows monitoring of intracranial pressure and } \\
\text { collection of CSF samples }\end{array}$ \\
\hline $\begin{array}{l}\text { Lesion thought to be fungal in } \\
\text { origin but not responding to } \\
\text { antifungal therapy }\end{array}$ & $\begin{array}{l}\text { Surgical open biopsy or stereotactic } \\
\text { biopsy }\end{array}$ & Choice of approach depends on lesion location and size \\
\hline $\begin{array}{l}\text { Acute hemispheric stroke with } \\
\text { mass effects (ischemic or } \\
\text { hemorrhagic) }\end{array}$ & $\begin{array}{l}\text { Hemicraniectomy for large middle- } \\
\text { cerebral-artery stroke; hematoma } \\
\text { aspiration }\end{array}$ & $\begin{array}{l}\text { Skull bone removal (craniectomy) reduces intracranial pressure } \\
\text { and brain herniation }\end{array}$ \\
\hline $\begin{array}{l}\text { Mycotic aneurysm with } \\
\text { subarachnoid hemorrhage }\end{array}$ & $\begin{array}{l}\text { Coiling or surgical aneurysm, parent- } \\
\text { artery occlusion, or both }\end{array}$ & $\begin{array}{l}\text { Severe subarachnoid hemorrhage- induced arterial vasospasm } \\
\text { may require catheter angioplasty }\end{array}$ \\
\hline
\end{tabular}

